\title{
The Effect of Various Cognitive-Motor and Motor- Motor Tasks on the Motor and Cognitive Function of Patients Multiple Sclerosis: A Randomized Controlled Trial
}

\author{
Arash Abasnia \\ University of Tehran \\ Mehdi Shahbazi ( $\square$ shahbazimehdi@ut.ac.ir) \\ University of Tehran https://orcid.org/0000-0002-2148-4150 \\ Elahe Arab Ameri \\ University of Tehran
}

\section{Research article}

Keywords: motor control, gait, balance, multiple sclerosis, secondary task, cognition, information processing speed, Stroop, rehabilitation

Posted Date: September 18th, 2020

DOI: https://doi.org/10.21203/rs.3.rs-56668/v1

License: (c) (1) This work is licensed under a Creative Commons Attribution 4.0 International License.

Read Full License 


\section{Abstract}

Background: $85 \%$ of multiple sclerosis patients suffer from impaired motor function and $65 \%$ from cognitive impairment. Therefore The main purpose of this study is to improve the cognitive and motor function of patients using dual tasks.

Methods: The study samples were 60 women with MS (20-50 years) with an Expanded Disability Status Scale 2-4 and the duration of the period (10-14 years) who were divided into 4 groups and for eight weeks each week including two sessions 45 They practiced dual cognitive, motor, and individual training for 45 to 60 minutes. Data were analyzed by composite variance analysis at significance level of $P<0.05$.

Results: The results of compound analysis analysis showed that the training of cognitive-motor tasks improved the significance of motor function $(p=0.0005)$ and cognitive performance $(p=0.0001)$ compared to the other three groups.

Conclusion: The results of this study suggest that the physical benefits of training are always superior to cognitive changes. Dual-task training can be considered a good way to improve cognitive and cognitive function.

Trial registration: This study was registered in the Iranian Clinical Trial Center with the number IRCT20200812048384N1, http://www.irct.ir/trial/50300, registered 26 August 2020.

\section{Background}

Multiple Sclerosis (MS) is a type of progressive degenerative disease of the myelin sheath in Central nervous system (CNS) [1]. Despite advances in medical science in recent years, the MS has already had no definitive treatment, and most of the available treatments lead to a reduction in symptoms or a reduction in the rate of disease progression, so it prevents the rapid diagnosis and detection of disease and its timely control of it. Symptoms range from a wide range that can be referred to as weakness of muscles, Ataxia, spasm, vision disorders, sensory disorders, and cognitive disorders [2], as well as reducing mobility abilities and fatigue from the underlying problems. More than $85 \%$ of these patients suffer from walking and non - balance problems [4], and when these problems are accompanied by a cognitive task [5]. As a result, physical and cognitive functions are important parts of a successful motor function. However, the impairment of the physical domain [6,7] and cognitive domains [8], observed in MS, may lead to related disorders that may result in the inability to manage daily activities.

Integration between movement and cognitive domains is measured using the dual-task (DT) model, which refers to the ability to perform two simultaneous tasks $[8,9]$. In particular, these two tasks refer to motor tasks (e. g. task math) and an additional task at the same time, from any range of cognition (e. g. a mathematical task)or movement type (e.g., holding a glass of water) [10]. Researchers recommend that the placement of individuals in the interest - sharing situations (i.e., DT) helps them plan appropriate control strategies [11]. DT's performance is related to most of the everyday life activities and the inability 
of patients to perform efficient tasks may have different consequences. Recently, several studies have been conducted on healthy elderly people [12-15] or in people with mental illnesses (e.g., Parkinson's disease) [16] using DT exercises, whose results showed improvement of balance and walking. indirectly, the benefits of walking and learning separately indicate that performance in these tasks can be improved in patients with targeted interventions. Specifically, DT training in patients with dementia and Parkinson's disease show that by training purposeful DT, motor and cognitive impairment can be minimized.

The aim of this study was to investigate the effect of DT training on motor and cognitive function in MS patients. In particular, we sought to investigate the effect of cognitive-motor (CM), motor-motor (MM) and single task (ST) on motor components (walking, balance), cognitive component (speed and accuracy of information processing) and psychological components (quality of life (QOL), anxiety and depression). In particular, we hypothesized for overall improvement in motor and cognitive function in both DT conditions after $16 \mathrm{DT}$ training sessions. In addition, previous studies have suggested that participants who undergo MM DT training do not differ significantly in motor factors from the other three groups and ST and MC groups.

\section{Method}

\section{Trial design}

This single-blind, randomized, and controlled trial, which was conducted at Iran MS Society rehabilitation centre in Tehran, Iran, lasted for 8 weeks starting in October and ending in November 2019. Participants were blinded to the type of exercise and the intervention group. This study included three steps: 1 . Preintervention measurements 2. 8-week DT and ST training for case groups, And the control group resumed their normal lives, and 3. Post-intervention measurements.

Prior to the intervention, participants were tested for berg balance tests (BBT), time-to-up and go (TUG) tests, quality of life questionnaires, anxiety and depression, and Stroop information processing tests. Participants were randomly divided into four groups of 15 (Fig. 1).

\section{Participants}

\section{Figure 1 Participant Flow Diagram}

The participants in this study were patients with MS in Tehran province. Using G-Power software with a test power of 0.8 , the effect size was 0.8 and the error level was 0.05 , the sample size was 60 people. Sampling was also available and 60 eligible participants were selected from 78 participants. Conditions for entering the study range are 20-50 years, EDSS (2-4), disease period (10-14), recurrence in recent months (at least one month), non-addiction to drugs and alcohol, no fractures and joint and movement problems and The ability to recognize English colours in the Stroop test. Participants were informed that their participation in the study was voluntary and confidential and that they could leave the study at any time without any problems. 


\section{Study intervention}

After the pre-test and identifying the groups, our intervention began. group (1), CM tasks that simultaneously performed a cognitive task (such as counting backwards) and a motor task (such as walking over an obstacle); (2) the dual MM tasks performed by two simultaneous motor tasks (such as walking and wearing a heater), the ST group (3) performing only walking training, and (4) the control of their daily lives. Participants practised for 8 weeks, including 2 sessions per week and 45-60 minutes per session, and finally, the pre-test tests were used again in the post-test period. It should be noted that the basic movement training in all 3 experimental groups (or walking), which was constant in 3 training groups, includes the following 10 items that have been borrowed from functional gait assessment (FGA):

Going to safety, changing speed, walking with a head turn, walking with a back turn, walking 180 turns, stepping on obstacles, walking with minimal support, walking blindfolded, walking backwards, and stairs.

In the CM DT training group, FGA was associated with cognitive training that included counting backward numbers, counting coins and paper money, texting, multiplying and dividing numbers, and naming the solar months from beginning to end. Participants changed from simple to difficult.

In the MM DT Training Group, FGA merged with the most commonly used daily movement training, such as tying coat buttons, wearing clothes, tying watches, hanging clothes on hangers, and carrying a cup of water.

In this study, the ST force practiced only the FGA movement task, and the control group lived a normal life during the intervention. Before each training session, the instructors explained and performed the training, and all of the proposed training were complexes in terms of complexity, tailored to the individual abilities of the participants.

It should be noted that all the trains were performed in the form of circular training for more efficiency, and each person went to the next movement after the specified time of each movement (30 seconds of activity and 45 seconds of rest until the start of the next movement). As the participants' level of performance increased, the ratio of training time to rest increased.

\section{Randomization and blinding}

In this study, participants were randomly divided into 4 groups according to the days they could come to the gym. It should be noted that none of the factors involved in the study were blind.

\section{Measurement}

Demographic data (age, height, weight, disease duration, EDSS, and BMI) were collected. Before the test, each participant was assessed for height and weight (Table 1). All research was conducted in a fixed location and the instructors were constant throughout the study. 
The walking speed was measured using the TUG field test, which is a simple test to

assess people's mobility and requires static and dynamic balance. The balance was measured using a berg balance test, which is a clinical trial to assess static and dynamic balance, especially in neurological patients. Patients' quality of life was measured with the MSIS-29 questionnaire, which contains 29 questions, the first 20 questions of physical impact and the last 9 questions of the psychological impact of MS on the patient. Participants' anxiety and depression were also measured by the hospital's anxiety and depression questionnaire. This 4-item selection questionnaire was designed to measure mood swings, especially anxiety and depression. Finally, patient information processing was measured by an online strop test. The online test has 20 questions. The 15 names and color questions are conflict with each other, and the 5 name and color questions are the same. And the output of this test is presented in the form of a table that shows the number of correct questions and the time of the test.

Table 1

Average and standard deviation related to the individual characteristics of the subjects

\begin{tabular}{|lllll|}
\hline group & MM & CM & C & ST \\
Variable & Mean \pm SD & Mean \pm SD & Mean \pm SD & Mean \pm SD \\
\hline age (year) & $4 / 5 \pm 45$ & $6.45 \pm 40$ & $8.12 \pm 45$ & $34 / 7 \pm 46$ \\
\cline { 1 - 3 } height $(\mathrm{cm})$ & $68 / 7 \pm 162.46$ & $32 / 5 \pm 164.6$ & $21 / 6 \pm 163$ & $87 / 6 \pm 163.2$ \\
\cline { 1 - 4 } weight $(\mathrm{kg})$ & $02 / 5 \pm 64.33$ & $21 / 7 \pm 64$ & $45 / 6 \pm 64.5$ & $32 / 5 \pm 63.4$ \\
\hline Duration (years) & $56 / 2 \pm 12.6$ & $2 \pm 12.4$ & $04 / 2 \pm 12.8$ & $2 / 2 \pm 12.13$ \\
\hline EDSS & $\pm 2.38 / 0$ & $\pm 2.78 / 0$ & $\pm 2.67 / 0$ & $\pm 3.28 / 0$ \\
\hline BMI & $(5.4) 29.3$ & $26.1(3.9)$ & $27.3(4.5)$ & $27.5(4.5)$ \\
\hline
\end{tabular}

\section{Ethical considerations}

This study's protocols were reviewed and approved by the research ethics committee faculty of physical education and sport sciences at the University of Tehran in Iran (Registration no.:

IR.UT.SPORT.REC.1398.049). The study's protocol was also registered in the Iranian Registry of Clinical Trials (Registration no.: IRCT20200812048384N1). All the tests and measurements were carried out at the Iranian MS Association. As well, all the participants were provided with related informed consent forms which were both completed and signed by participants in person.

\section{Statistical Analysis}

IBM spss25 software was used for statistical analysis. To determine the mean and standard deviation, descriptive statistics were used to normalize the data from Azen Shapiro-Wilk and to compare between groups, assuming that the composite analysis of variance was parametric and in all cases the significance level was $p<0.05$. 


\section{Results}

There were no side effects during the study, and most of the participants who participated in the training from the beginning completed the study.

The results of the composite variance analysis test in the post-test phase indicate that there is a significant difference between the test results in the post-test $\left[F_{(21,56)}=8.531, p<0.05\right]$. The results of the intergroup comparison also show that there is a significant difference between the groups in the post-test walking speed scores $\left[\mathrm{F}_{(3,56)}=17.333, \mathrm{p}<0.05\right]$. As you can see in diagram1; All three post-test training groups showed better walking speed than the control group $(p<0.05)$. Also, CM

training group compared to the ST training group; Indicates better walking speed in the post-test $(\mathrm{p}<$ 0.05). Also, there was no significant difference between the two groups of $\mathrm{CM}$ and $\mathrm{MM}$ training and the two groups of MM and ST in the post-test walking speed scores $(p<0.05)$.

Digram 1. Comparison diagram of the dependent variable of the path between the four control groups, MC task, MM task and ST in the pre-test and post-test stages.

The results of the composite variance analysis test in the post-test phase showed that there was a significant difference between the test results in the post-test $[F(21,56)=8,531, p<0.05]$. There is also a result of intergroup comparison showing a significant difference between groups in post-test equilibrium scores $[F(3,56)=44,840, p<0.05]$. As you can see in diagram2; All three post-test training groups showed better balance than the control group $(p<0.05)$. CM training group also showed better post-test balance than the other two training groups $(p<0.05)$. However, there was no significant difference between the two groups of MM and ST training in post-test balance scores $(p<0.05)$.

Digram 2. Comparison Chart dependent variable balance between the four groups: control, MC tasks, task MM and ST on pre- and post-tests.

The results of the composite variance analysis test in the post-test phase showed that there was a significant difference between the test results in the post-test $[F(21,56)=8,531, p<0.05]$. Also in the intergroup comparison; There was no significant difference between the groups in the post-test accuracy, processing, and information scores $[F(3.56)=0.178, p>0.05]$. As you can see in diagram 3 ; Comparing the two groups of $\mathrm{CM}$ and $\mathrm{MM}$ training with the post-test control group, there was a significant difference in the accuracy of information processing and scoring scores compared to the control group $(p<0.05)$. However, no significant difference was observed when compared to an ST group with the control group in post-test.

Diagram3. Comparison diagram of the dependent variable of processing accuracy between the four control groups, CM, MM task and ST in the pre-test and post-test stages

Also, in comparison with the $\mathrm{CM}$ training group compared to the other two training groups, a significant difference was observed in the post-test $(p<0.05)$. Also, there was no significant difference between the 
two groups

of MM and ST training in accuracy scores, processing, post-test information $(p<0.05)$.

The results of the composite variance analysis test in the post-test phase showed that there was a significant difference between the test results in the post-test $[F(21,56)=8,531, p<0.05]$. Also in the intergroup comparison; There was no significant difference between the groups in the scores of, information processing speed post-test $[F(3,56)=0.178, p>0.05]$. As you can see in Fig. 4; All three posttest practice groups did not show a significant difference in the speed, information processing speed scores compared to the control group $(\mathrm{p}<0.05)$. Also, compared to the $\mathrm{CM}$ training group compared to the other two training groups, no significant difference was observed in the post-test $(p<0.05)$. Also, there was no significant difference between the two groups of MM and ST training in post-test information processing speed scores $(p<0.05)$.

Diagram 4. Comparison diagram of the dependent variable of processing speed between four control groups, CM task, MM task and ST in pre-test and post-test stages.

The results of the composite variance analysis test in the post-test phase showed that there was a significant difference between the test results in the post-test $[F(21,56)=8,531, p<0.05]$. Also, the result of the intergroup comparison shows a significant difference between groups in post-test quality of life scores. [ $F(3,56)=44,840, p<0.05]$. As you can see in diagram5; All three post-test training groups showed a better quality of life score than the control group $(p<0.05)$. Also, the CM training group showed better quality of life scores in the post-test than the other two training groups $(p<0.05)$. Also, the CM training group showed better life quality scores in the pre-test $(p<0.05)$ than two other training groups. but there were no significant differences in the quality of life in the pre-test $(p<0.05)$.

Diagram 5 - Comparison chart of the dependent variable of quality of Life between the four control groups, CM, MM and ST in the pre-test and post-test stages.

The results of the composite variance analysis test in the post-test phase showed that there was a significant difference between the test results in the post-test $[F(21,56)=8,531, p<0.05]$. Also, the result of the intergroup comparison shows a significant difference between groups in post-test anxiety depression scores. $[F(3,56)=44,840, p<0.05]$. As you can see in diagram 6 ; The two post-test $C M$ and post-test groups showed a better depression anxiety score than the control group $(p<0.05)$. But this was not the case in the MM group ( $p$ <.05). The CM training group also showed better depression anxiety scores in the post-test than the other two training groups $(p<0.05)$. However, no significant differences were observed between the two groups of MM training and ST in anxiety scores Post-test depression was not observed $(p<0.05)$.

Diagram 6. Comparison chart of the dependent variable of anxiety, the depression between the four control groups, CM task, MM task and ST in the pre-test and post-test stages. 


\section{Discussion}

The aim of this study was to investigate the effect of a CM and MM, DT training program on the motor function of MS patients. For this purpose, we examined the effects of 8 weeks of DT training with secondary motor and cognitive tasks in a sample of MS individuals to examine the effect of DT on the motor and cognitive function of MS patients. In particular, we evaluated performance at walking speed, balance, information processing, quality of life, and anxiety and depression in ST and DT conditions. The present study sought to develop previous work in several ways. First, limited studies used DT as a training method. Second, using the other form of DT is a MM training on the patients that this type of exercise is more important to patients with brain loss - spinal cord injuries, elderly people and Parkinson's patients, which is, of course, important that the results are contradictory and we intended to make comparisons between the two practice methods to achieve a specific result. The results of the present study showed that an 8-week program improved the condition of the CM training group in all factors compared to the other three groups. The MM training group and the ST group did not differ significantly from each other except in the information processing (accuracy) section in the other factors studied. Also, the three training groups had a significant difference in balance, walking speed and quality of life compared to the control group. In terms of information processing accuracy, the CM group and the MM group were better than the control group. Also, the CM group was better than the MM group, but the MM group did not have a significant difference with the ST group. All four groups did not have a significant difference in post-test information speed and processing. In the following, we will review and compare several studies in this field.

In a study titled The Impact of DT training on MS Patients Sossnof et al. (2017), they found that dual CM training improved the walking speed of MS patients but did not affect patients' cognitive function. The study is present because as we saw in Digram 1, there was no significant difference between CM and MM group in walking speed. Also, the CM training group in the accuracy of information processing accuracy was better than any other three groups that contradict the study of Sossnof et al. (2017) [17].

In another study, Manjezi et al. (2017) measured the effect of dual-task Balance training on the postural performance of MS patients and found that there was no significant difference between the balance CM and ST groups, which is inconsistent with the present study, as shown in Diagram 2. We have shown that the group of post-test CM training showed more improvement than the other 3 groups [18].

Mercan et al. (2016) measured the effect of CM and MM tasks with open and closed eyes on the balance of MS patients and concluded that both forms of training improve patients' balance, but MM training with Closed eyes are more useful than other methods, and these results are somewhat at odds with the present study because we found in the balance discussion that the $\mathrm{CM}$ group achieved a significant improvement in balance over other groups [19].

Finally, the justification for the lack of difference between the two groups of MM and ST can be attributed to 10 items of FGA training. During the intervention, patients reported that FGA walking training was very cognitive for them and that walking was not done automatically. For example, in walking training over the 
patient's obstacles, they needed to identify the distance to the obstacle, adjust the step, and choose a support base, which in turn imposed a lot of cognitive load on the participants.

Also, the better performance of the CM group in the factors of quality of life, anxiety and depression can be attributed to the interesting and challenging cognitive training.

The reason why this research is inconsistent with our study can be due to the intervention, disability and type of disease of patients, gender and differences in the form of exercises that are debatable.

Doing two tasks at the same time requires attention to resources and integration of executive performance [20],Therefore, we may speculate that MS patients who have enrolled in our DT practice may learn to manage their attention between these two tasks [21]. In fact, two independent streams of visual information, one related to the walking task and the other related to the secondary task, must be synchronized [22]. Participants in CM, DT training may improve task management and coordination skills to perform two tasks simultaneously [23]. Therefore, our results suggest that CM, DT training may alter the DT strategy and lead to better and more efficient integration of the two tasks [24].

\section{Conclusion}

As a result, our results suggest that 8 weeks of CM, DT training can improve motor, cognitive, quality of life, and anxiety, and depression in patients in relation to ST and MM -DT groups. In no other case did the processing of information differ significantly. However, further studies using larger sample sizes appear to be necessary to expand this study and integrate gender. Our results as a DT training, including secondary cognitive tasks, may improve patients' motor and cognitive function. To understand the role of certain types of training, focusing on DT function for people with MS, it may be helpful for older people to develop specific strategies and guidelines for specific interventions.

\section{Limitations}

Several potential limitations of this study should be considered. First, all patients in the study were female and the sex was not integrated. Second, the relatively small size of the sample does not allow us to generalize our results to a larger population. The use of FGA testing as a basic motor exercise in all 3 groups, this test could affect the results of the study due to the difficulty of the items and the need for cognitive activity to perform. And last but not least, the cost of dual homework in all three groups. Although these findings support the predominant effect of our training protocol on physical aspects, the benefits of cognitive training are not clear.

\section{Abbreviations}

MS: multiple sclerosis; DT: dual task; CM: cognitive -motor; MM: motor-motor; ST: single task; TUG: timed up and go; BBT: berg balance test; QOL: quality of life; FGA: functional gait assessment CNS: central nervous system; BMI: Body Mass Index.EDSS: Expanded Disability Status Scale 


\section{Declarations}

\section{Acknowledgment}

I would like to thank all the patients participating in this research and the respected authorities of my community. We thank the province of Tehran for helping us with this research.

\section{Authors' contributions}

AA and MSH were responsible for the title and design of the study. AA was also responsible for collecting and analyzing data. EA was the first in responsible for the drafts, which were reviewed by all the authors. All authors have read and approved the manuscript.

\section{Funding}

The study was not funded by any organization or association.

\section{Availability of data and materials}

The datasets used and/or analyzed during the current study are available from the corresponding author on reasonable request.

\section{Availability of data and materials}

Supplemental material for this article is available online.

\section{Ethics approval and consent to participate}

Patients have a right to privacy that should not be infringed without informed consent. And the patients give written informed consent for publication that approved by the research ethics committee faculty of physical education and sport sciences at the University of Tehran in Iran (Registration no.:

IR.UT.SPORT.REC.1398.049). This study was registered in the Iranian Clinical Trial Center with the number IRCT20200812048384N1, http://www.irct.ir/trial/50300, registered 26 August 2020.

\section{Consent for publication}

Not applicable.

\section{Competing interests}

The authors declare that they have no competing interests.

\section{Author details}

${ }^{1}$ Research Fellow, Faculty of Physical Education and Sport Sciences, University of Tehran, Tehran, Iran 
${ }^{2}$ Department of Motor Behavior, Faculty of Physical Education and Sport Sciences, University of Tehran, Tehran, Iran

\section{References}

1. Gmeindl L, Courtney SM. in Multiple Sclerosis. 2013;26(1):57-70.

2. Kister I, Bacon TE, Chamot E, Salter AR, Cutter GR, Kalina JT, et al. Natural history of multiple sclerosis symptoms. Int J MS Care. 2013;15(3):146-58.

3. Butchard-Macdonald E, Paul L, Evans JJ. Balancing the Demands of Two Tasks: An Investigation of Cognitive-Motor Dual-Tasking in Relapsing Remitting Multiple Sclerosis. J Int Neuropsychol Soc. 2018;24(3):247-58.

4. Hamilton F, Rochester L, Paul L, Rafferty D, O'leary CP, Evans JJ. Walking and talking: an investigation of cognitive-motor dual tasking in multiple sclerosis. Mult Scler J. 2009;15(10):121527.

5. Brustio PR, Magistro D, Liubicich ME. Changes in temporal parameters during performance of the Step Test in older adults. Gait Posture. 2015;41(1):217-21.

6. Magistro D, Candela F, Brustio PR, Liubicich ME, Rabaglietti E. A longitudinal study on the relationship between aerobic endurance and lower body strength in italian sedentary older adults. $J$ Aging Phys Act. 2015;23(3):444-51.

7. Park HL, O'Connell JE, Thomson RG. A systematic review of cognitive decline in the general elderly population. Int J Geriatr Psychiatry. 2003;18(12):1121-34.

8. Agmon M, Belza B, Nguyen HQ, Logsdon RG, Kelly VE. A systematic review of interventions conducted in clinical or community settings to improve dual-task postural control in older adults. Clin Interv Aging. 2014;9:477.

9. Chu Y, Tang P, Peng Y, Chen H. Meta-analysis of type and complexity of a secondary task during walking on the prediction of elderly falls. Geriatr Gerontol Int. 2013;13(2):289-97.

10. Mcisaac TL, Lamberg EM, Muratori LM. Building a Framework for a Dual Task Taxonomy. $2015 ; 2015$.

11. Peruzzi A, Cereatti A, Della Croce U, Mirelman A. Effects of a virtual reality and treadmill training on gait of subjects with multiple sclerosis: a pilot study. Mult Scler Relat Disord. 2016;5:91-6.

12. Agmon M, Kodesh E, Kizony R. The effect of different types of walking on dual-task performance and task prioritization among community-dwelling older adults. Sci World J. 2014;2014.

13. Kitazawa K, Showa S, Hiraoka A, Fushiki Y, Sakauchi H, Mori M. Effect of a dual-task net-step exercise on cognitive and gait function in older adults. J Geriatr Phys Ther. 2015;38(3):133-40.

14. Pichierri G, Wolf P, Murer K, de Bruin ED. Cognitive and cognitive-motor interventions affecting physical functioning: a systematic review. BMC Geriatr. 2011;11(1):29.

15. Wollesen B, Voelcker-Rehage $C$. Training effects on motor-cognitive dual-task performance in older adults: A systematic review. Eur Rev Aging Phys Act. 2014;11(1):5-24. 
16. Yogev-Seligmann G, Giladi N, Brozgol M, Hausdorff JM. A training program to improve gait while dual tasking in patients with Parkinson's disease: a pilot study. Arch Phys Med Rehabil. 2012;93(1):176-81.

17. Sosnoff JJ, Wajda DA, Sandroff BM, Roeing KL, Sung J, Motl RW. Dual task training in persons with Multiple Sclerosis: A feasability randomized controlled trial. Clin Rehabil. 2017;31(10):1322-31.

18. Monjezi S, Negahban H, Tajali S, Yadollahpour N, Majdinasab N. Effects of dual-task balance training on postural performance in patients with Multiple Sclerosis: A double-blind, randomized controlled pilot trial. Clin Rehabil. 2017;31(2):234-41.

19. Mercan F, Kara B, Tiftikcioglu BI, Mercan E, Sertpoyraz FM. Effects of motor-motor and motorcognitive tasks on balance in patients with multiple sclerosis. Mult Scler Relat Disord [Internet]. 2016;7:85-91. Available from: http://dx.doi.org/10.1016/j.msard.2016.03.015

20. Leone C, Feys P, Moumdjian L, D'Amico E, Zappia M, Patti F. Cognitive-motor dual-task interference: A systematic review of neural correlates. Neurosci Biobehav Rev [Internet]. 2017;75:348-60. Available from: http://dx.doi.org/10.1016/j.neubiorev.2017.01.010

21. Wongcharoen S, Sungkarat S, Munkhetvit P, Lugade V, Silsupadol P. Home-based interventions improve trained, but not novel, dual-task balance performance in older adults: a randomized controlled trial. Gait Posture. 2017;52:147-52.

22. Beurskens R, Bock $O$. Does the walking task matter? Influence of different walking conditions on dual-task performances in young and older persons. Hum Mov Sci [Internet]. 2013;32(6):1456-66. Available from: http://dx.doi.org/10.1016/j.humov.2013.07.013

23. Silsupadol P, Shumway-Cook A, Lugade V, van Donkelaar P, Chou L-S, Mayr U, et al. Effects of singletask versus dual-task training on balance performance in older adults: a double-blind, randomized controlled trial. Arch Phys Med Rehabil. 2009;90(3):381-7.

24. Gregory MA, Boa Sorte Silva NC, Gill DP, McGowan CL, Liu-Ambrose T, Shoemaker JK, et al. Combined Dual-Task Gait Training and Aerobic Exercise to Improve Cognition, Mobility, and Vascular Health in Community-Dwelling Older Adults at Risk for Future Cognitive Decline1. J Alzheimer's Dis. 2017;57(3):747-63.

\section{Figures}




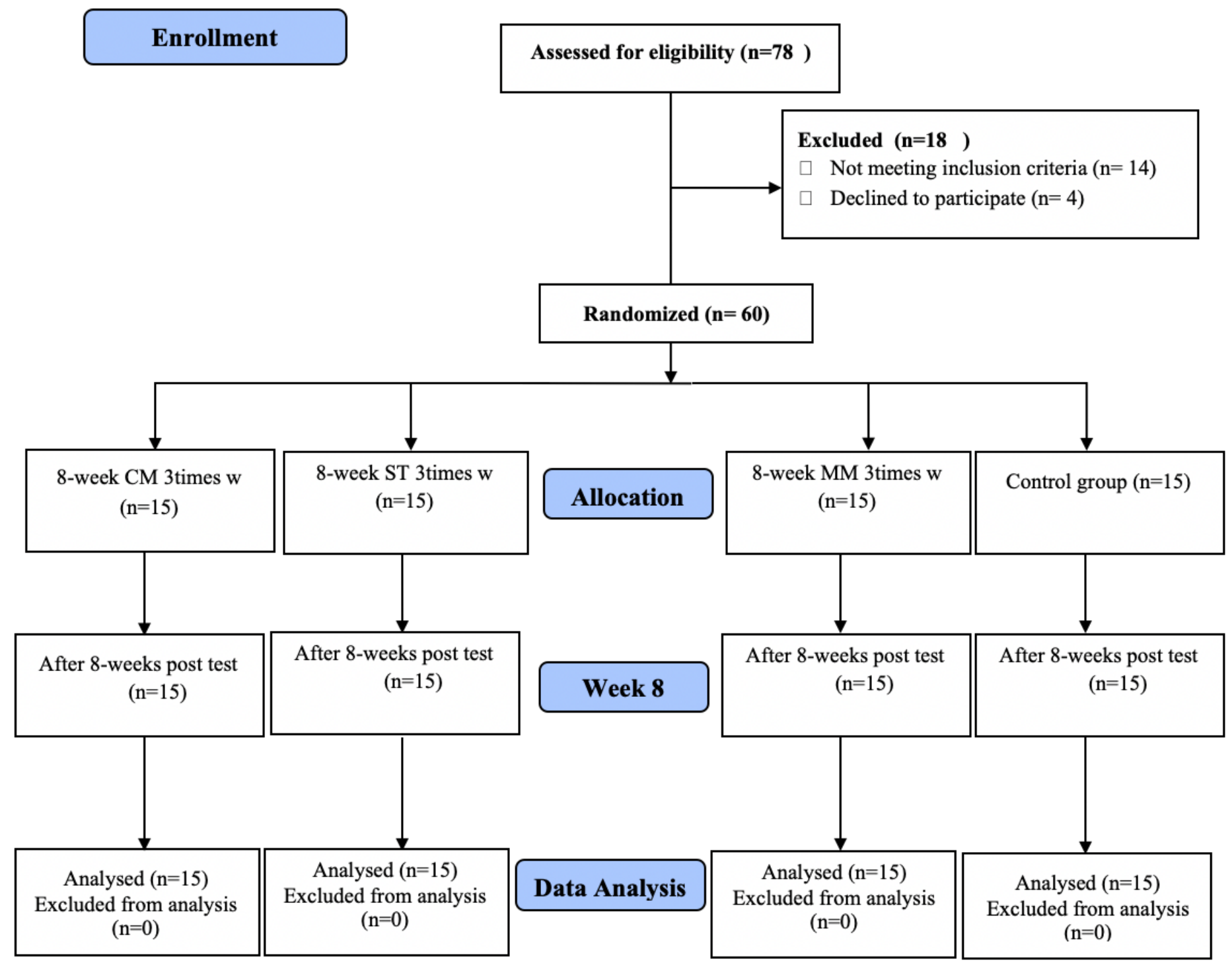

Figure 1

Participant Flow Diagram 


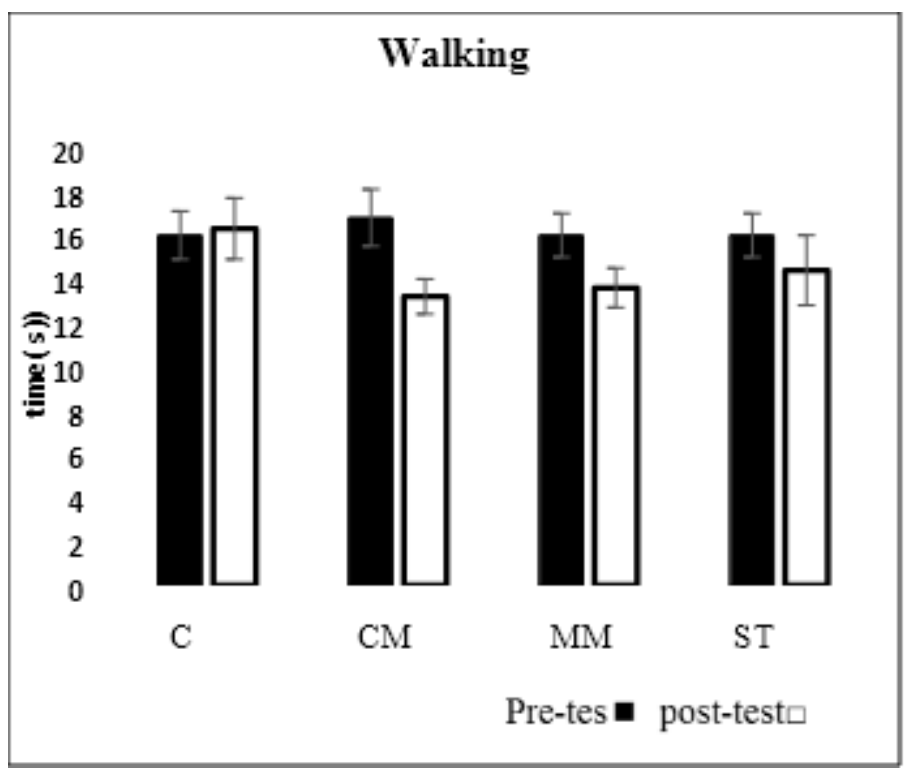

Figure 2

Digram 1. Comparison diagram of the dependent variable of the path between the four control groups, MC task, MM task and ST in the pre-test and post-test stages.

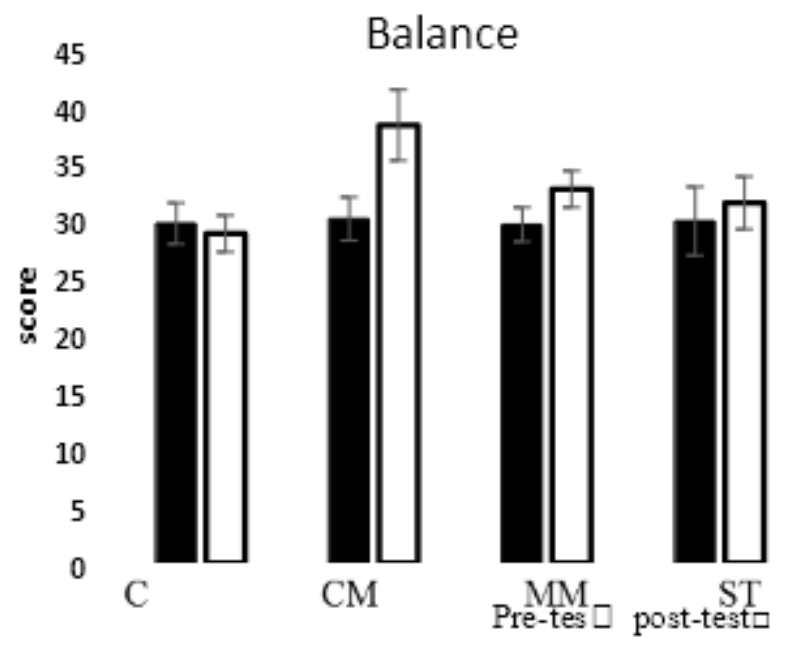

Figure 3

Digram 2. Comparison Chart dependent variable balance between the four groups: control, MC tasks, task MM and ST on pre- and post-tests. 


\section{Processing accuracy}

25

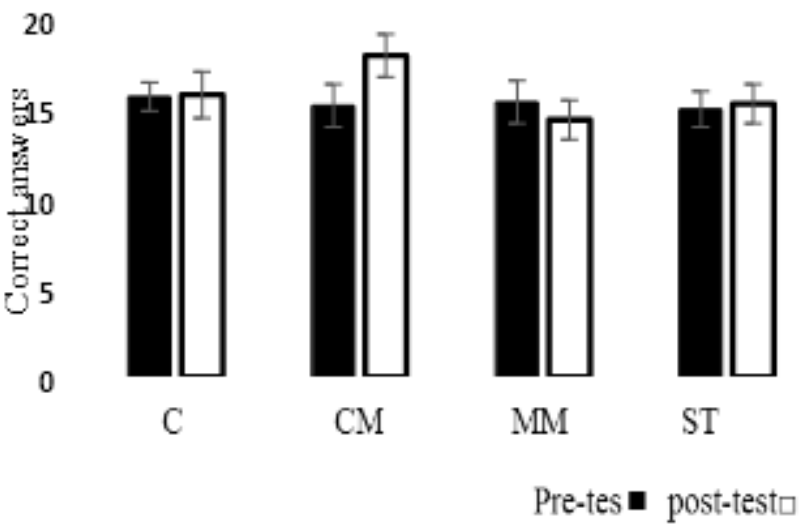

Figure 4

Diagram3. Comparison diagram of the dependent variable of processing accuracy between the four control groups, CM, MM task and ST in the pre-test and post-test stages

information processing speed

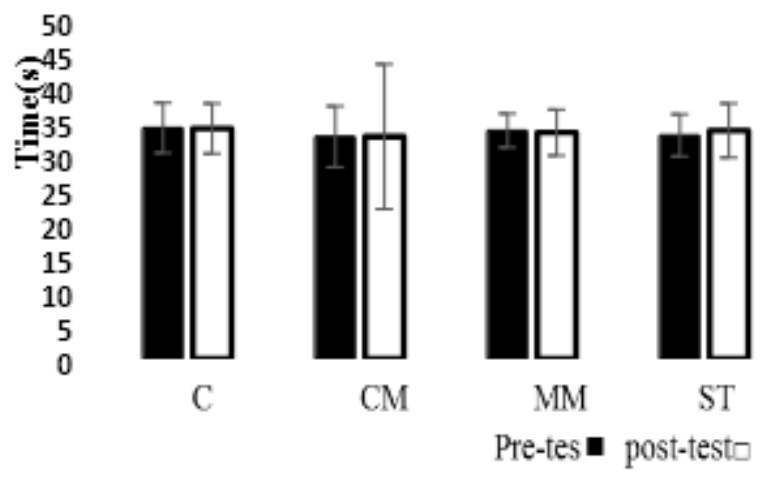

Figure 5

Diagram 4. Comparison diagram of the dependent variable of processing speed between four control groups, CM task, MM task and ST in pre-test and post-test stages. 


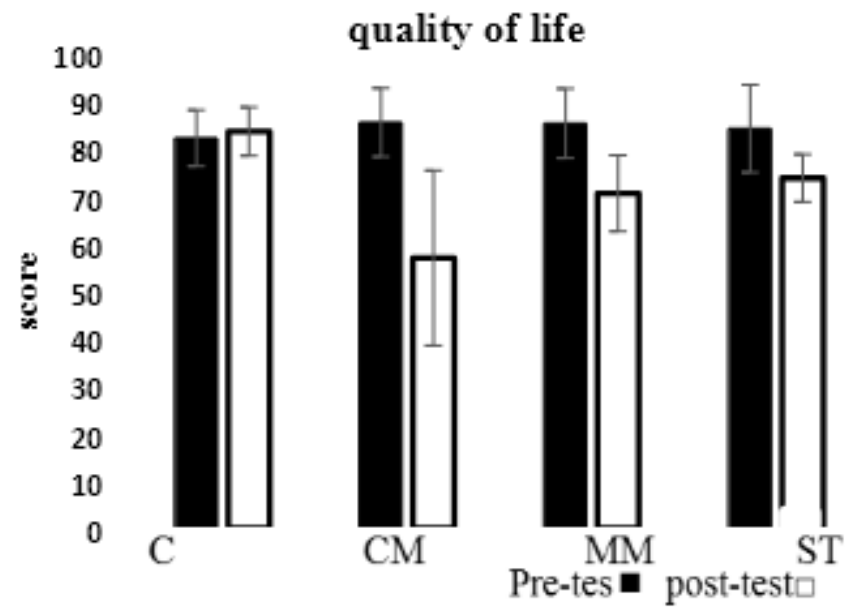

Figure 6

Diagram 5 - Comparison chart of the dependent variable of quality of Life between the four control groups, CM, MM and ST in the pre-test and post-test stages.

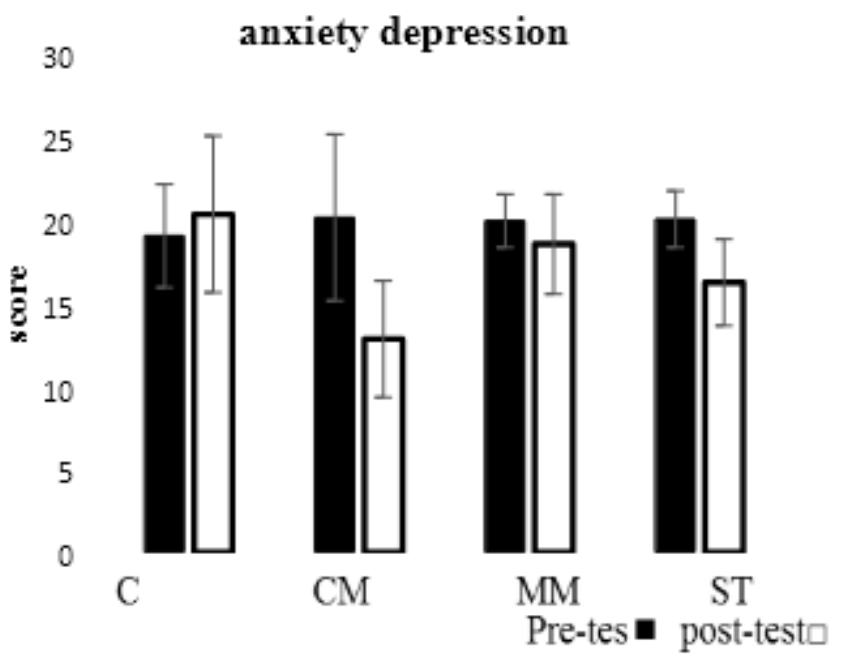

Figure 7

Diagram 6. Comparison chart of the dependent variable of anxiety, the depression between the four control groups, CM task, MM task and ST in the pre-test and post-test stages.

\section{Supplementary Files}

This is a list of supplementary files associated with this preprint. Click to download.

- CONSORT2010Checklist.doc

- CONSORT2010FlowDiagram1.doc 